\title{
The SF-36 and 6-Minute Walk Test as Predictors of Complications After Major Surgery, Clinical Impact
}

\author{
J. Straatman ${ }^{1}$ D. L. van der Peet ${ }^{1}$
}

Published online: 9 July 2015

(C) Société Internationale de Chirurgie 2015

To Editor,

With interest we read the prospective observational study regarding the SF-36 and 6-min Walk Test as predictors of complications following major surgery by Awdeh et al. published in the World Journal of Surgery [1]. The group subjected all patients scheduled to undergo thoracotomy, sternotomy, or upper abdominal laparotomy to a pre-operative assessment, using the SF-36 health survey and 6-min walk test. The pre-operative 6-min walk test was found to be a useful predictor of postoperative complications.

As the walking distance was associated with the complication grade, we wonder how this can be implemented in a clinical setting? Also, does an impaired 6-min walking test justify a differentiated surgical approach, and which cut-off in walking distance is recommended? Taking into account that a linear regression model was used without the input of other variables that may be used as predictors (e.g., co-morbidity, SF-36).

Quality control algorithms are gaining increasing interest in surgical practice. A standardized evidence-based approach should be used in pre-operative assessment, surgical techniques, pathological examination, and postoperative monitoring in order to obtain optimal outcomes. The manuscript by Awdeh et al. underwrites the importance of assessment of the pre-operative patient [1]. The optimal assessment and consequences may be incorporated in a

J. Straatman

je.straatman@vumc.nl

1 Department of Gastrointestinal Surgery, VU University Medical Center, De Boelelaan 1117, 7F 020,

1081 HV Amsterdam, The Netherlands quality control algorithm, ensuring optimal patient care and allocation of patients to facilities that address their respective needs.

Such a quality control algorithm should not only assess the pre-operative patient, but also aim to early assess and diagnose complications in the postoperative patient. The implementation of a standardized postoperative quality control algorithm, aimed at early diagnosis and treatment of major complications after major abdominal surgery, can decrease morbidity and mortality [2,3]. Currently our project group is focusing on setting up a prospective randomized clinical trial with standardized postoperative measurements of C-reactive protein, in order to facilitate early diagnosis of major complications and as a safe discharge criterion (www.precious-trial.nl).

\section{Compliance with Ethical Standards}

Conflict of interest We declare that none of the authors have conflicts of interest to disclose.

\section{References}

1. Awdeh H, Kassak K, Sfeir P, Hatoum H, Bitar H, Husari A (2015) The SF-36 and 6-minute walk test are significant predictors of complications after major surgery. World J Surg 39(6):1406-1412. doi:10.1007/s00268-015-2961-4

2. Straatman J, Cuesta MA, Gisbertz SS, van der Peet DL (2014) Value of a step-up diagnosis plan: CRP and CT-scan to diagnose and manage postoperative complications after major abdominal surgery. Rev Esp Enferm Dig 106(8):515-521

3. Rivers E, Coba V, Whitmill M (2008) Early goal-directed therapy in severe sepsis and septic shock: a contemporary review of the literature. Curr Opin Anaesthesiol 21(2):128-140 